\title{
Ciência brasileira: impactos para muito além do fator de impacto
}

\author{
Brazilian science: impacts far beyond the impact factor
}

Sigmar de Mello Rode', Germana Barata²

1Universidade Estadual Paulista. São José dos Campos, São Paulo, Brasil. ORCID: 0000-0002-4261-4217. sigmarrode@uol.com.br ${ }^{2}$ Autora para correspondência. Universidade Estadual de Campinas. Campinas, São Paulo, Brasil. ORCID: 0000-0001-6064-6952. germana@unicamp.br

De acordo com a Coordenação de Aperfeiçoamento de Pessoal de Ensino Superior (Capes), o sistema Qualis Periódicos "é o conjunto de procedimentos utilizados pela instituição para estratificação da qualidade da produção intelectual dos programas de pós-graduação"1. Ele foi concebido para atender a necessidades específicas do sistema de avaliação e é baseado nas informações fornecidas pelos programas de pós-graduação. Como resultado, disponibiliza uma lista com a classificação dos periódicos utilizados pelos programas para a divulgação da sua produção no quadriênio avaliado².

Em 2009, quando a Capes decidiu pela criação de um número maior de estratos para poder reclassificar os periódicos, foi proposta uma escala decrescente segundo o valor do Fator de Impacto (FI): A1, A2, B1, B2, B3, B4, B5 e C.
A então nova classificação havia sido elaborada com base na mediana do Fl das revistas, obtidas junto ao Journal Citation Reports (JCR) e calculado anualmente pela base de dados ISI Web of Knowledge. Para o cálculo da mediana foi elaborada uma lista das revistas em que cada área da Capes publica. De posse desta lista, bem como dos respectivos Fl, foi calculada a mediana para cada área e construída a nova estratificação, que dependendo da área pôde incorporar outro índice de citação como por exemplo o Cites per Doc $^{3}$ (citações por documentos).

Em 2019, a Capes divulgou proposta de Qualis provisório, na avaliação de meio termo, com uma nova classificação: A1, A2, A3, A4, B1, B2, B3, B4 e C, utilizando basicamente Scopus (CiteScore), Web of Science (FI) - comerciais - e Google Scholar (índice h5), mas deixando de fora a base SciELO, que indexa revistas brasileiras de acesso aberto. 
Uma das poucas vantagens da proposta foi que o periódico terá um único Qualis, estabelecido pela chamada área-mãe ${ }^{4}$. Essa nova proposta gerou grande manifestação contrária e bem fundamentada na academia ${ }^{5,6}$ e que aponta para a desvalorização das revistas científicas nacionais com o fortalecimento de critérios de internacionalização.

Em 1955, um pesquisador americano chamado Eugene Garfield, hoje considerado um dos fundadores da bibliometria e da cienciometria, propôs um índice para avaliar a relevância das publicações indexadas no então Institute of Scientific Information (ISI), denominado Fator de Impacto (FI) para auxiliar bibliotecários a identificar publicações de modo mais eficiente e menos subjetivo. Desde então, o Fl firmou-se como meio de avaliação dos periódicos nas mais variadas instâncias, sendo calculado anualmente pelo ISI (atual Clarivate Analytics) para as revistas indexadas em sua base de dados, e publicado pelo JCR.

O Fl é exclusivo do JCR e para o seu cálculo, leva-se em consideração o número de citações recebidas pelos artigos publicados no periódico nos dois anos anteriores à avaliação, dividido pelo número de artigos publicados no mesmo período. Entre outros problemas bem documentados ${ }^{8}$, o $\mathrm{Fl}$ é calculado somente pelo que ocorre nos periódicos indexados na base, pode ser manipulado e existe a questão de que um artigo pode ser citado não necessariamente por ser bom, mas para ser contestado devido a algum erro nos métodos empregados ou na interpretação dos resultados experimentais. Nesse indicador, a centralidade é dada à revista científica como um todo, apesar de influenciar a avaliação de artigos e, consequentemente, o impacto que autores produziram individualmente, mesmo sabendo que cerca de $20 \%$ das revistas receberam $80 \%$ das citações e cerca de $23 \%$ dos artigos de uma mesma revista sequer recebem uma única citação e $51 \%$ recebem de 1 a 5 citações apenas 9 .

A Declaração de San Francisco sobre Avaliação da Pesquisa ${ }^{8}$ (DORA) recomenda, entre outras coisas, não usar métricas baseadas em periódicos, tais como o $\mathrm{Fl}$, como uma medida indireta da qualidade de artigos de pesquisa individuais, para avaliar as contribuições de um pesquisador, ou em decisões de contratação, promoção ou financiamento.

Apesar de inúmeras instituições, pesquisadores e entidades acadêmicas serem signatárias da Declaração de DORA, vemos que o FI continua presente e forte no delineamento de políticas científicas. Mais do que uma ferramenta para indicar relevância de publicações, o Fator de Impacto acabou influenciando fortemente políticas científicas, rankings universitários, produtividade acadêmica e políticas editoriais de revistas científicas como as brasileiras.

Parte das limitações do Qualis está na dificuldade de usar uma mesma régua para áreas do conhecimento distintas. Claramente, revistas multidisciplinares acabam prejudicadas, como é o caso dos Anais da Academia Brasileira de Ciências (AABC) que, a cada mudança no Qualis, experimenta diminuição ou aumento no número de submissões para determinadas áreas ${ }^{10}$. Isso valeria também para cursos de pós-graduação multidisciplinares, que publicam em uma ampla gama de revistas de áreas-mãe distintas.

Uma das limitações do Qualis está na supervalorização de indexadores internacionais anteriormente citados com baixa representatividade de revistas brasileiras e da área de humanidades, tradicionalmente menos internacionalizada. São apenas 314 revistas brasileiras (apenas 157 no JCR) ${ }^{11}$ e que se destacam por serem publicações consideradas de "mainstream", ou seja, tratarem de temáticas de relevância internacional e não nacional ou local. Com isso, valoriza-se a produção internacional em detrimento da produção nacional. O mesmo ocorre na área das ciências exatas e biomédicas em relação às humanidades.

O Qualis deixou de fora a principal base de indexação de revistas científicas brasileiras, a coleção SciELO, mesmo que parte dela esteja representada nas duas bases internacionais. As revistas da coleção SciELO têm reconhecida relevância para impacto nacional e 
local, com maior número de citações para as áreas de ciências sociais, humanas, psiquiatria, psicologia, economia e negócios, além das ciências agrárias ${ }^{12} \mathrm{e}$ da saúde pública e coletiva ${ }^{13}$, todas com destaque para citações de outros autores brasileiros.

Mais do que ampliar a cobertura de periódicos nas bases Scopus e Web of Science, é preciso ampliar os indicadores de impacto das publicações brasileiras na pós-graduação. Iniciando pela inclusão da coleção SciELO, bem como de periódicos considerados relevantes e não indexados - os quais correspondem a $60 \%$ das revistas registradas por doutores no currículo Lattes ${ }^{14}$ e de outros tipos de publicações acadêmicas relevantes para as humanidades, como capítulos de livros e livros, e de indicadores complementares como a altmetria.

As chamadas métricas alternativas (altmetria) propõem medir a atenção que artigos e documentos científicos receberam em plataformas online, como as redes sociais, blogs, notícias jornalísticas, documentos governamentais, Wikipedia entre outras. Além de tirar a centralidade das revistas científicas, colocando o valor no artigo individualmente, a altmetria lembra os inúmeros usos que uma publicação pode ganhar na era digital, seja compondo documentos governamentais, como o noticiário, os verbetes que auxiliam a compreensão de conceitos ou postagens nas redes sociais que amplificam a divulgação de resultados de pesquisa no Facebook, Twitter ou em vídeos do YouTube. Se as publicações acadêmicas são capazes de contribuir para o debate social e políticas públicas, para fomentar aulas e trabalhos educativos, além de gerar as tão valorizadas citações, então a comunicação acadêmica produzida nos programas de pós-graduação, em sentido amplo, estão cumprindo com sua missão.

A cada novo sistema Qualis, a comunidade pede por aperfeiçoamentos. Mas, nos parece ainda válida a conclusão de Rita de Cássia Barradas Barata quando avaliou o sistema em 2016: "é necessário combinar diferentes fontes de informação e indicadores de impacto, buscando minimizar as limitações inerentes a cada um, e, finalmente, é importante desenvolver um sistema que permita a comparação entre diferentes áreas e elimine as contradições atualmente existentes no sistema"15.

Está na hora de repensarmos o peso da avaliação Qualis como determinante de políticas editoriais e como fator de direcionamento da produção científica. É preciso valorizar o artigo em si, pela quantidade de leituras e citações, independente da base de indexação de dados.

\section{Referências}

1. CAPES. Ministério de Educação. Considerações sobre Qualis Periódiocos e os critérios para a estratificação e uso dos mesmos na avaliação. 2016. [acesso em 18 de novembro de 2019]. Disponível em: https://capes.gov.br/images/documentos/Qualis_ periodicos_2016/Qualis_Servi\%C3\%A7o_Social_.pdf

2. Rocha-e-Silva M. O novo Qualis, ou a tragédia anunciada. Clinics. 2009; 64(1):1-4. doi: 10.1590/S1807-59322009000100001

3. Andriolo A, Souza AFM, Farias AQ,Barbosa AJA, França Netto AS, Hernandez AJ et al. Classificação dos Periódicos no sistema QUALIS da CAPES - A mudança dos critérios é URGENTE! Arq Bras Endocrinol Metab. 2010; 54(1):1-3. doi: 10.1590/S0004$\underline{27302010000100002}$

4. Capes. CAPES melhora ferramentas de avaliação da pósgraduação. [Internet]. 2019. [acesso em 18 de novembro de 2019]. Disponível em: https://www.capes.gov.br/36-noticias/9730capes-melhora-ferramentas-de-avaliacao-da-pos-graduacao

5. ABRASCO. Fórum de Coordenadores de Pós-Graduação em Saúde Coletiva. Apreciação da Proposta de Qualis Periódico referência. [Internet]. 2017. [acesso em 18 de novembro de 2019] Disponível em: https://www.abrasco.org.br/site/ coordenadoresdepos/wp-content/uploads/sites/2/2019/08/CartaABRASCO-sobre-Proposta-Qualis-Referencia.pdf

6. Fundação Oswaldo Cruz. Carta aberta sobre a proposta do Qualis Periódicos. [Internet]. 2019. [acesso em 18 de novembro de 2019]. Disponível em: http://periodicos.fiocruz.br/sites/ default/files/anexos/carta_FECF_criterios-Qualis-Capes_v2_ Fitosassina_22082019_0.pdf 
7. Beuren IM, Souza JC. Em busca de um delineamento de proposta para classificação dos periódicos internacionais de contabilidade para o Qualis CAPES. R Cont Fin. 2008;19(46):44-58. doi: 10.1590/S1519-70772008000100005

8. DORA. San Francisco Declaration on Research Assessment. [Intenet]. 2012. [acesso em 18 de novembro de 2019]. Disponível em: https://sfdora.org/read

9. Gargouri Y, Hajjem C,Lariviere V, Gingras Y, Carr L, Brody T et al. Self-Selected or Mandated, Open Access Increases Citation Impact for Higher Quality Research. PLoS One. 2010;5(10): e13636. doi: $\underline{10.1371 / \text { journal.pone.0013636 }}$

10. Kellner AWA. The Qualis system: a perspective from a multidisciplinary journal. An Acad Bras Ciênc. 2017; 89(3):13391342. doi: $10.1590 / 0001-37652017893$

11. ABEC. Relatório de fator de impacto das revistas brasileiras 2017-2018. Disponível em: https://www.abecbrasil.org.br/ arquivos/fator_impacto17-18.pdf

12. Mugnaini R, Noyons E, Packer AL. Fluxo de citações internacional: fontes de informação para avaliação de impacto científico no Brasil. Anais do 6o Encontro Brasileiro de Bibliometria e Cientometria: a ciência em rede. [Internet]. 2018. [acesso em 15 de novembro de 2019]. Disponível em: http://ebbc. inf.br/ebbc6/docs/6EBBC2018v2018_07_27.pdf

13. Packer AL. Indicadores de centralidade nacional da pesquisa comunicada pelos periódicos de Saúde Coletiva editados no Brasil Ciênc Saúde Coletiva. 2015;20(7):1983-1995. doi: 10.1590/1413$\underline{81232015207.07122015}$

14. Mugnaini R, Dmaceno RJP, Digiampietri LA, mena-chalco JP. Panorama da produção científica do Brasil além da indexação: uma análise exploratória da comunicação em periódicos. Transinformação, 2019; 31:e190033. doi: 10.1590/2318$0889201931 \mathrm{e} 190033$

15. Barata RCB. Dez coisas que você deveria saber sobre o Qualis. RBPG. 2016;13(1):1-18. doi: 10.21713/2358-2332.2016.v13.947 